\title{
Producción y calidad de pepino (Cucumis sativus L.) bajo condiciones de invernadero usando dos sistemas de poda
}

\author{
Production and quality of cucumber (Cucumis sativus L.) \\ under greenhouse conditions using two pruning systems \\ Jesús López-Elías ${ }^{1}$, Julio C. Rodríguez ${ }^{1}$, Marco A. Huez L. ${ }^{1}$, Sergio Garza O. ${ }^{1}$, \\ José Jiménez L. ${ }^{1}$, Edgar I. Leyva E. ${ }^{1}$
}

\section{RESUMEN}

En México, la producción de hortalizas bajo invernadero se ha incrementado significativamente durante los últimos años, siendo importante la producción de pepino (Cucumis sativus L.), ocupando el 10\% de la superficie invernada. Para evaluar el efecto del sistema de poda en la producción y calidad de pepino partenocárpico se realizó un estudio en un invernadero localizado en el Campo Agrícola Experimental de la Universidad de Sonora, durante el período comprendido de septiembre de 2009 a enero de 2010. Los tratamientos fueron tres híbridos de pepino (Camán, Esparón y Modán) y dos sistemas de poda (Descuelgue a un tallo, sin eliminar el punto apical del tallo principal y Descuelgue a dos tallos, eliminando al inicio del descuelgue el punto apical del tallo principal pero dejando dos brazos laterales). El diseño del experimento fue de bloques completos al azar en arreglo factorial, con cuatro repeticiones. Se evaluaron los días a floración, e inicio de cosecha, la producción (número de frutos por planta, peso del fruto y rendimiento), al igual que la calidad de la producción (longitud, diámetro y firmeza del fruto). En el ciclo agrícola de otoño-invierno el manejo agronómico del cultivo de pepino en invernaderos sin calefacción se puede realizar con el descuelgue del cultivo a un tallo, debido al retraso en el crecimiento de los nuevos brotes al eliminar el punto apical del tallo principal, al igual que las bajas temperaturas retardan el crecimiento de los brotes secundarios; además de que dicha técnica requiere de menor mano de obra. No se observó precocidad por parte de alguno de los híbridos, al igual que por el sistema de poda, iniciando la floración a los 33 días después de la siembra y la cosecha a los 69 días. El número de frutos por planta en el híbrido Esparón, con descuelgue a un tallo, fue mayor respecto de Camán y Modán, con 17,7 frutos planta ${ }^{-1}$, recomendándose su implementación por presentar también bajo porcentaje de flores masculinas y menor cuateo. El peso del fruto, al igual que los parámetros de calidad, estuvo dentro de los estándares establecidos para pepino tipo americano, con un peso promedio de $330 \mathrm{~g}$ en la categoría Fancy, longitud de $23,2 \mathrm{~cm}$, diámetro de $5,0 \mathrm{~cm}$ y firmeza de $4,8 \mathrm{~kg}$.

Palabras clave: Cucumis sativus, entrenamiento, invernadero, pepino, rendimiento.

\section{ABSTRACT}

In Mexico, vegetable production under greenhouse conditions has increased significantly in recent years; cucumber (Cucumis sativus L.) production is important, representing $10 \%$ of greenhouse crops. To evaluate the effect of pruning system on yield and quality of parthenocarpic cucumbers, a study was carried out in a greenhouse at the Agricultural Experimental Station of the Sonora University, during the period September 2009 to January 2010. The treatments were three cucumber hybrids (Caman, Esparon, and Modan), and two pruning systems (without removing the growing point of the main stem, and the growing point of the main stem removed and two lateral branches near the top of the plant allowed to grow, resulting in two branches growing downward). The experimental design was a completely randomized block design with factorial arrangement, with four replications. Days to flowering and to the beginning of harvest, commercial production (fruit number per plant, fruit weight and yield), as well as production quality (fruit length, diameter and firmness) were evaluated. During autumn-winter production, the agronomic management of cucumber in unheated greenhouses should be done without removing the growing point of the main stem, since the growth of new branches is delayed when the apical point of the main stem is removed and low temperatures retard the growth of secondary branches; this technique also requires less labor. Precocity was not observed in any of the hybrids, or due to pruning system; flowering began at 33 days after sowing and harvest at 69 days. The fruit number per plant in Esparon was greater with respect to Caman and Modan when the growing point of the main stem was not removed, with 17.7 fruit plant $^{-1}$; its implementation is recommended, since it also presented a low percentage of male flowers and fewer double fruit. Fruit weight and quality parameters were within the standards established for American type cucumbers, with an average weight of $330 \mathrm{~g}$ in Fancy category, $23.2 \mathrm{~cm}$ length, $5.0 \mathrm{~cm}$ diameter and $4.8 \mathrm{~kg}$ firmness.

Key words: Cucumber, Cucumis sativus, greenhouse, pruning, yield.

1 Universidad de Sonora, Departamento de Agricultura y Ganadería. Carretera a Bahía de Kino, Km. 21. Hermosillo, Sonora. México. E-Mail: lopez_eliasj@guayacan.uson.mx 


\section{Introducción}

La agricultura como toda actividad humana implica la explotación del medio natural. En concreto, la agricultura intensiva pretende producir el máximo con la menor ocupación posible del suelo, para lo cual se recurre a una serie de técnicas con el objetivo de forzar la producción. Un ejemplo de este tipo de producción lo tenemos en el cultivo bajo invernadero. Este se orienta a obtener el más alto rendimiento, a costa de aislarlo de las condiciones naturales mediante el forzado del cultivo a través de técnicas de climatización (calefacción, humidificación, iluminación, etcétera) y técnicas culturales (fertirrigación, sustratos, etcétera) para rentabilizar al máximo la ocupación del terreno. Esta rentabilización implica una mejora en la utilización de los recursos naturales, agua y suelo (Antón, 2004).

La implementación de la producción hortícola en invernadero disminuye el riesgo de la producción, incrementa la rentabilidad del sector productivo; además de que genera fuente de trabajo, disminuye la contaminación ambiental y los daños a la salud (Grijalva y Robles, 2003).

Aunque la industria de los invernaderos nació y se desarrolló en Europa, para principios de los 80 empezó a tomar impulso en América, especialmente en Canadá y algunas regiones de Estados Unidos. En México, aunque desde los 70 nacen en el altiplano, con flores (sobre todo en los estados de Ciudad de México y de Morelos), es a finales de los 90 cuando comienzan a desarrollarse en forma importante en la producción intensiva de hortalizas, pasando de 1998 al 2006 (tan solo ocho años) de 600 a más de 6.500 hectáreas. Sonora ocupa el tercer sitio con 707 hectáreas construidas y 180 por construir, siendo superado solo por Sinaloa, Baja California y Jalisco (Garza y Molina, 2008).

La cubierta predominante en la agricultura protegida en México, con $47 \%$ es la de plástico, $50 \%$ de malla sombra, vidrio $2 \%$ y $1 \%$ de otro tipo de material (Destenave, 2007).

El tomate ocupa el $70 \%$ del volumen producido en invernadero, el pepino $10 \%$, el pimiento $5 \%$ y otros cultivos concentran un 15\% (Destenave, 2007).

El pepino se considera originario de la India, siendo domesticado en Asia y de ahí introducido a Europa, para posteriormente ser llevado a América por Cristóbal Colón. Los tipos más comunes de pepino son el americano, el europeo, el del este medio, el holandés y el pepino oriental (Wehner y Maynard, 2003)

La demanda de pepino en los Estados Unidos de Norteamérica ha tenido un crecimiento sin precedentes en los últimos años. La importación creció de 394.107 toneladas en 2002 a 459.242 toneladas en 2007, es decir, un incremento del $16,5 \%$ en solo cinco años (FAOSTAT, 2010). De este volumen de importación 361.721 toneladas proceden de México, siendo el principal país exportador de pepino hacia los Estados Unidos (MercaNET, 2007).

En el 2009 en México se sembraron alrededor de 14.600 ha de pepino con rendimiento de $30,0 \mathrm{tha}^{-1}$ como media de producción. En el estado de Sonora ese mismo año se programó una superficie de siembra de alrededor de 570 ha, ocupando el quinto lugar a nivel nacional. Con respecto a la Costa de Hermosillo, en el 2009 se sembraron alrededor de 220 ha con un rendimiento promedio de $18,2 \mathrm{tha}^{-1}$ (SIAP, 2010).

La producción de pepino en invernadero en el noroeste de México ha sido un éxito, al obtenerse buenos rendimientos con una sola duración del ciclo, siendo esta de 108 días en invierno, lo que da oportunidad de realizar dos siembras al año prolongando así la ventana de producción (Hernández, 2006). Bajo condiciones de invernadero, la producción de pepino es de 2 a 9 veces más que en campo abierto, dependiendo del nivel tecnológico, el manejo y las condiciones climatológicas (FUMIAF, 2005), constituyendo asimismo una alternativa a la diversificación de cultivos en invernadero.

La forma de poda más comúnmente usada en pepino bajo condiciones de invernadero consiste en eliminar por abajo de los 40 a $50 \mathrm{~cm}$ del tallo principal todos los brotes que salgan, al igual que las hojas y los frutos que se vayan formando. A partir de los 40 a $50 \mathrm{~cm}$ se eliminan todos los brotes laterales que aparecen en el tallo principal, dejando un fruto en cada axila, hasta que este alcance el alambre superior usado para el entrenado de la planta. Una vez que una o dos hojas se han desarrollado por arriba del alambre, el punto terminal del tallo principal es eliminado, dejando crecer libremente en el extremo superior de la planta dos brazos laterales, eliminando la yema terminal cuando la planta está cerca del suelo (Hochmuth, 2001).

En cuanto a la composición y calidad del producto final, las mismas dependen del genotipo, manejo agronómico del cultivo (densidad de plantación, fertilización, irrigación, al igual que 
el control de plagas y enfermedades), condiciones climáticas, estado de madurez a la cosecha y el método de cosecha (Kader, 1996).

El objetivo del presente trabajo de investigación fue evaluar la respuesta de tres híbridos de pepino americano, bajo dos sistemas de poda al descuelgue del cultivo en condiciones de invernadero.

\section{Materiales y Métodos}

El presente trabajo se desarrolló en un invernadero localizado en el Campo Agrícola Experimental del Departamento de Agricultura y Ganadería de la Universidad de Sonora, a los $29^{\circ} 00^{\prime} 48^{\prime \prime}$ lat N, $111^{\circ} 08^{\prime} 07^{\prime}$ ' long O y $150 \mathrm{msnm}$, durante el ciclo otoño-invierno 2009-2010.

El invernadero tiene una superficie de $225 \mathrm{~m}^{2}$, con cubierta de polietileno de 8 mil de espesor, pared húmeda, dos extractores y sin sistema de calefacción. El suelo es de textura franco arenosa y agua para riego con conductividad eléctrica de $0,57 \mathrm{dSm}^{-1} \mathrm{y}$ $\mathrm{pH}$ de 7,29.

El cultivo evaluado fue pepino (Cucumis sativus L.) partenocárpico, tipo americano, cuya siembra directa se realizó el 15 de septiembre de 2009. La densidad de población fue de 3,3 plantas $\mathrm{m}^{-2}$, a doble hilera separada $30 \mathrm{~cm}$, con distanciamiento de $40 \mathrm{~cm}$ entre plantas y distancia entre hileras de 1,5 m. Establecido el cultivo, su manejo fue de acuerdo a las prácticas habituales del productor de la región, llevándose a cabo aplicaciones preventivas y de control químico de insectos y enfermedades.

Los tratamientos evaluados fueron: a). Tres híbridos de pepino americano (Camán, Esparón y Modán) y b). Dos sistemas de poda (1. Descuelgue a un tallo, sin eliminar el punto apical del tallo principal y 2. Descuelgue a dos tallos, eliminando al inicio del descuelgue el punto apical del tallo principal pero dejando dos brazos laterales). En ambos sistemas de poda, la yema terminal se eliminó 1,2 $\mathrm{m}$ antes de alcanzar el suelo.

Las plantas fueron entrenadas a un solo tallo, sostenidas con hilo rafia de polipropileno sujeto a un alambre transversal que cruza el invernadero a una altura de $2,5 \mathrm{~m}$. Por abajo de $10 \mathrm{~s} 40 \mathrm{~cm}$ del tallo principal se eliminaron todos los brotes, al igual que las hojas y frutos. Posteriormente, en forma semanal se efectuaba la poda, eliminando todos los brotes laterales, dejando un fruto por axila, hasta que la planta alcanzó el alambre transversal. Una vez que se tuvieron dos hojas desarrolladas por arriba del alambre, se procedió con los tratamientos de poda, eliminando la yema terminal cuando la planta estaba a un metro del suelo. El riego se distribuyó a través de goteros de $2,0 \mathrm{~L} \mathrm{~h}^{-1}$, aplicando una lámina total de $60,5 \mathrm{~cm}$. La fertilización total fue de $300 \mathrm{~N}-180 \mathrm{P}-$ 260K-200Ca-25Mg, la cual se distribuyó a lo largo del ciclo del cultivo, aplicando dos veces por semana y ajustando de acuerdo a análisis foliares realizados quincenalmente.

El diseño experimental fue de bloques completos al azar con arreglo factorial, con seis tratamientos y cuatro repeticiones, con ocho plantas por repetición. El área experimental fue de $105 \mathrm{~m}^{2}$, dentro de la cual se establecieron 24 unidades experimentales de $3 \mathrm{~m}^{2}$ (2,0 $\mathrm{m}$ de largo por 1,5 $\mathrm{m}$ de ancho).

Las variables a evaluar fueron los días a floración, presencia de flores masculinas, cuateo de fruto, días a inicio de descuelgue y días a inicio de corte (estos dos últimos, considerando un $20 \%$ de presencia del evento referido); asimismo, se evaluó la producción comercial (frutos por planta, peso del fruto y rendimiento), al igual que la calidad del fruto (longitud, diámetro y firmeza). Para el peso del fruto en gramos se usó una balanza digital marca Avery Berkel, modelo 6405, con precisión de $5 \mathrm{~g}$. La longitud del fruto se determinó usando una cinta metálica, con precisión de $1 \mathrm{~mm}$. Para el diámetro del fruto en mm se usó un vernier digital marca Mitutoyo, modelo CD-6" CS, con precisión de 0,01 $\mathrm{mm}$. La firmeza del fruto en $\mathrm{kg}$ se determinó usando un penetrómetro marca Wagner, modelo FT 20, con precisión de $100 \mathrm{~g}$.

Una vez cosechados los pepinos se seleccionaron de acuerdo con las normas de calidad. Primero, se clasificaron por su grado de madurez; después por su tamaño, preferentemente de 20 a $30 \mathrm{~cm}$ de largo, de superficie cilíndrica lisa y recta, color verde oscuro y uniforme (sin amarilleos), considerando como producción comercial la de categoría Fancy.

Para el análisis de los datos obtenidos en el experimento se usó el paquete estadístico SAS 6.12 (SAS Institute Inc., 1996). Se realizó el análisis de varianza de los datos, obteniéndose también la prueba de rango múltiple de Duncan con nivel de probabilidad del 5\%.

\section{Resultados y Discusión}

Los días a floración no se vieron afectados por el híbrido, promediando 33 días desde la siembra hasta el inicio de la floración, sin diferencias significativas 
entre tratamientos (Cuadro 1). Esto coincide con Premalatha et al. (2006) quienes observaron que la floración en pepino se presentó a los $33.4 \pm 0.9$ días, sin diferencias tanto entre híbridos como entre los sistemas de poda al descuelgue del cultivo que fueron evaluados.

En cuanto a la presencia de flores masculinas (Cuadro 1), lo cual se considera una característica no deseable en pepino partenocárpico, se observaron diferencias significativas entre tratamientos, siendo el híbrido Esparón, con 2,5\%, el que presentó un menor porcentaje de flores masculinas; en tanto que el híbrido Camán, con 30,0\% de flores masculinas, fue el que presentó el mayor porcentaje de flores masculinas.

Para la variable cuateo de frutos (Cuadro 1), es decir, la presencia de dos frutos por axila, se observaron diferencias significativas entre tratamientos. El híbrido Esparón presentó el menor porcentaje de cuateo, con 2,5\%, mientras que Modán y Camán presentaron un $72,5 \%$ y $77,5 \%$, respectivamente.

Cuadro 1. Días a floración, presencia de flor masculina y cuateo de frutos en pepino (Cucumis sativus L.) bajo condiciones de invernadero.

\begin{tabular}{lccr}
\hline Tratamiento & $\begin{array}{c}\text { Floración } \\
(\mathrm{d})\end{array}$ & $\begin{array}{c}\text { Flor masculina } \\
(\%)\end{array}$ & $\begin{array}{c}\text { Cuateo } \\
(\%)\end{array}$ \\
\hline Camán & $33^{\mathrm{a}}$ & $30,0 \mathrm{a}$ & $77,5 \mathrm{a}$ \\
Esparón & $32^{\mathrm{a}}$ & $2,5 \mathrm{~b}$ & $2,5 \mathrm{~b}$ \\
Modán & $33^{\mathrm{a}}$ & $10,0 \mathrm{ab}$ & $72,5 \mathrm{a}$ \\
\hline
\end{tabular}

Medias seguidas por la misma letra no son significativamente diferentes $(P=0,05)$.

Como se observa en el Cuadro 2, los híbridos Camán y Modán mostraron mayor desarrollo al alcanzar dos días antes que el híbrido Esparón la altura del alambre usado para el tutoreo de la planta, ubicado a 2,5 metros de altura; sin embargo, en el análisis estadístico no se encontraron diferencias significativas entre híbridos, lo cual coincide con el estudio en variedades de pepino realizado por Té (2008), como entre el sistema de poda al descuelgue del cultivo (Cuadro 3).

La cosecha se realizó a los 14 días posteriores a la apertura de la flor (antesis), momento en el cual el fruto presentaba una longitud de $20-25 \mathrm{~cm}$. Aunque el número de días a cosecha (Cuadro 2) se presentó con tres y cuatro días de antelación en el híbrido Modán, con respecto a Esparón y Camán respectivamente, estadísticamente no se encontraron diferencias significativas entre híbridos como entre el sistema de poda al descuelgue del cultivo (Cuadro 3), resultados que coinciden con Hochmuth et al. (1996) quienes no encontraron diferencias significativas para la producción precoz entre cultivares de pepino.

Cuadro 2. Inicio al descuelgue de la planta y a cosecha en pepino (Cucumis sativus L.) bajo condiciones de invernadero, expresados en días.

\begin{tabular}{lcc}
\hline Tratamiento & $\begin{array}{c}\text { Inicio a descuelgue } \\
(\mathrm{d})\end{array}$ & $\begin{array}{c}\text { Corte } \\
(\mathrm{d})\end{array}$ \\
\hline Camán & $49 \mathrm{a}$ & $70 \mathrm{a}$ \\
Esparón & $51 \mathrm{a}$ & $69 \mathrm{a}$ \\
Modán & $49 \mathrm{a}$ & $66 \mathrm{a}$ \\
\hline
\end{tabular}

Medias seguidas por la misma letra no son significativamente diferentes $(P=0,05)$.

Cuadro 3. Inicio al descuelgue de la planta y a cosecha en pepino (Cucumis sativus L.) bajo condiciones de invernadero, expresados en días.

\begin{tabular}{lcc}
\hline Tratamiento & $\begin{array}{c}\text { Inicio a descuelgue } \\
(\mathrm{d})\end{array}$ & $\begin{array}{c}\text { Corte } \\
(\mathrm{d})\end{array}$ \\
\hline Camán $^{1}$ & $48 \mathrm{a}$ & $69 \mathrm{a}$ \\
Camán $^{2}$ & $50 \mathrm{a}$ & $71 \mathrm{a}$ \\
Esparón $^{1}$ & $51 \mathrm{a}$ & $70 \mathrm{a}$ \\
Esparón $^{2}$ & $50 \mathrm{a}$ & $69 \mathrm{a}$ \\
Modán $^{1}$ & $48 \mathrm{a}$ & $67 \mathrm{a}$ \\
Modán $^{2}$ & $49 \mathrm{a}$ & $65 \mathrm{a}$ \\
\hline $1 \quad$ representa descuelgue a un tallo y $^{2}$ representa descuelgue \\
\multicolumn{2}{c}{ a dos tallos. } \\
Medias seguidas por la misma letra no son significativamente
\end{tabular}
diferentes $(P=0,05)$.

Los cortes dieron inicio el día 9 de noviembre, con un ritmo de recolección semanal. El número de frutos por planta, al igual que el peso del fruto y el rendimiento no se vieron afectados por el híbrido. Como se observa en el Cuadro 4, aunque el número de frutos por planta fue mayor en el híbrido Esparón con 16,8 frutos planta $^{-1}$, repercutiendo ello en el rendimiento del cultivo con un incremento superior al 6\%, estadísticamente no se encontraron diferencias significativas entre híbridos. Para las variables peso del fruto y rendimiento, los resultados coinciden con los obtenidos por Hochmuth et al. (1996) y Té (2008), quienes evaluando cultivares de pepino no encontraron diferencias significativas para dichas variables, coincidiendo asimismo con Té (2008), quien menciona que el peso del fruto en pepino americano fluctúa de 300 a 400 gramos. 
Del sistema de poda al descuelgue del cultivo (Cuadro 5), únicamente para la variable número de frutos por planta se observaron diferencias significativas entre tratamientos, siendo el híbrido Esparón con descuelgue a un tallo el que presentó mayor número de frutos, con 17,7 frutos planta ${ }^{-1}$; mientras que Camán con la misma forma de descuelgue presentó el menor número de frutos, con 14,6 frutos planta ${ }^{-1}$. La no diferencia significativa entre los sistemas de poda al descuelgue del cultivo coincide con Premalatha et al. (2006), lo cual se debe a que la producción en los tallos laterales es menor que en el tallo principal (Hochmuth, 2001), al igual que al retraso en el crecimiento de los nuevos brotes al eliminar el punto apical del tallo principal; además de que, durante el invierno, las temperaturas bajas (Figura 1) retrasaron el crecimiento del cultivo, minimizando el efecto del descuelgue.

Aunque con mayor número de frutos por planta en el híbrido Esparón, estadísticamente no se obtuvo mayor rendimiento, lo cual se debe principalmente a que los frutos son cosechados antes de alcanzar la madurez fisiológica y como su peso cambia rápidamente de un día a otro, el rendimiento depende de la fecha de corte (Staub et al., 2009).
Cuadro 4. Número de frutos por planta, peso del fruto (g) y rendimiento $\left(\mathrm{kg} \mathrm{m}^{-2}\right.$ y cajas $\mathrm{m}^{-2}$ ) en pepino (Cucumis sativus L.), categoría Fancy, bajo condiciones de invernadero.

\begin{tabular}{lcccc}
\hline Tratamiento & $\begin{array}{c}\text { Frutos } \\
\text { planta }^{-1}\end{array}$ & $\begin{array}{c}\text { Peso fruto } \\
(\mathrm{g})\end{array}$ & $\begin{array}{c}\text { Rendimiento } \\
\left(\mathrm{kg} \mathrm{m}^{-2}\right)\end{array}$ & $\begin{array}{c}\text { Rendimiento } \\
(\text { cajas ha } \\
\mathrm{z}\end{array}$ \\
\hline Camán & $15,1 \mathrm{a}$ & $337 \mathrm{a}$ & $16,3 \mathrm{a}$ & $6.751 \mathrm{a}$ \\
Esparón & $16,8 \mathrm{a}$ & $324 \mathrm{a}$ & $17,3 \mathrm{a}$ & $7.248 \mathrm{a}$ \\
Modán & $15,0 \mathrm{a}$ & $328 \mathrm{a}$ & $15,8 \mathrm{a}$ & $6.499 \mathrm{a}$ \\
\hline
\end{tabular}

z cajas de $25 \mathrm{~kg}$.

Medias seguidas por la misma letra no son significativamente diferentes $(P=0,05)$.

Cuadro 5. Número de frutos por planta, peso del fruto (g) y rendimiento $\left(\mathrm{kg} \mathrm{m}^{-2}\right.$ y cajas $\mathrm{m}^{-2}$ ) en pepino (Cucumis sativus L.), categoría Fancy, bajo condiciones de invernadero.

\begin{tabular}{|c|c|c|c|c|}
\hline Tratamiento & $\begin{array}{l}\text { Frutos } \\
\text { planta }^{-1}\end{array}$ & $\begin{array}{c}\text { Peso fruto } \\
(\mathrm{g})\end{array}$ & $\begin{array}{l}\text { Rendimiento } \\
\quad\left(\mathrm{kg} \mathrm{m}^{-2}\right)\end{array}$ & $\begin{array}{l}\text { Rendimiento } \\
\left(\text { cajas ha }^{-1}\right)^{\mathrm{z}}\end{array}$ \\
\hline Camán ${ }^{1}$ & $14,6 b$ & $345 a$ & $16,3 a$ & $6.695 a$ \\
\hline Camán² & $15,5 \mathrm{ab}$ & $329 a$ & $16,3 \mathrm{a}$ & $6.806 \mathrm{a}$ \\
\hline Esparón ${ }^{1}$ & $17,7 \mathrm{a}$ & $330 \mathrm{a}$ & $18,5 \mathrm{a}$ & $7.840 \mathrm{a}$ \\
\hline Esparón ${ }^{2}$ & $15,8 \mathrm{ab}$ & $318 \mathrm{a}$ & $16,0 \mathrm{a}$ & $6.656 a$ \\
\hline Modán ${ }^{1}$ & $14,9 \mathrm{ab}$ & $330 \mathrm{a}$ & $16,0 \mathrm{a}$ & $6.514 \mathrm{a}$ \\
\hline Modán ${ }^{2}$ & $15,1 \mathrm{ab}$ & $325 \mathrm{a}$ & $15,5 \mathrm{a}$ & $6.483 \mathrm{a}$ \\
\hline
\end{tabular}

1 representa descuelgue a un tallo $\mathrm{y}^{2}$ representa descuelgue a dos tallos.

z cajas de $25 \mathrm{~kg}$.

Medias seguidas por la misma letra no son significativamente diferentes $(P=0,05)$.

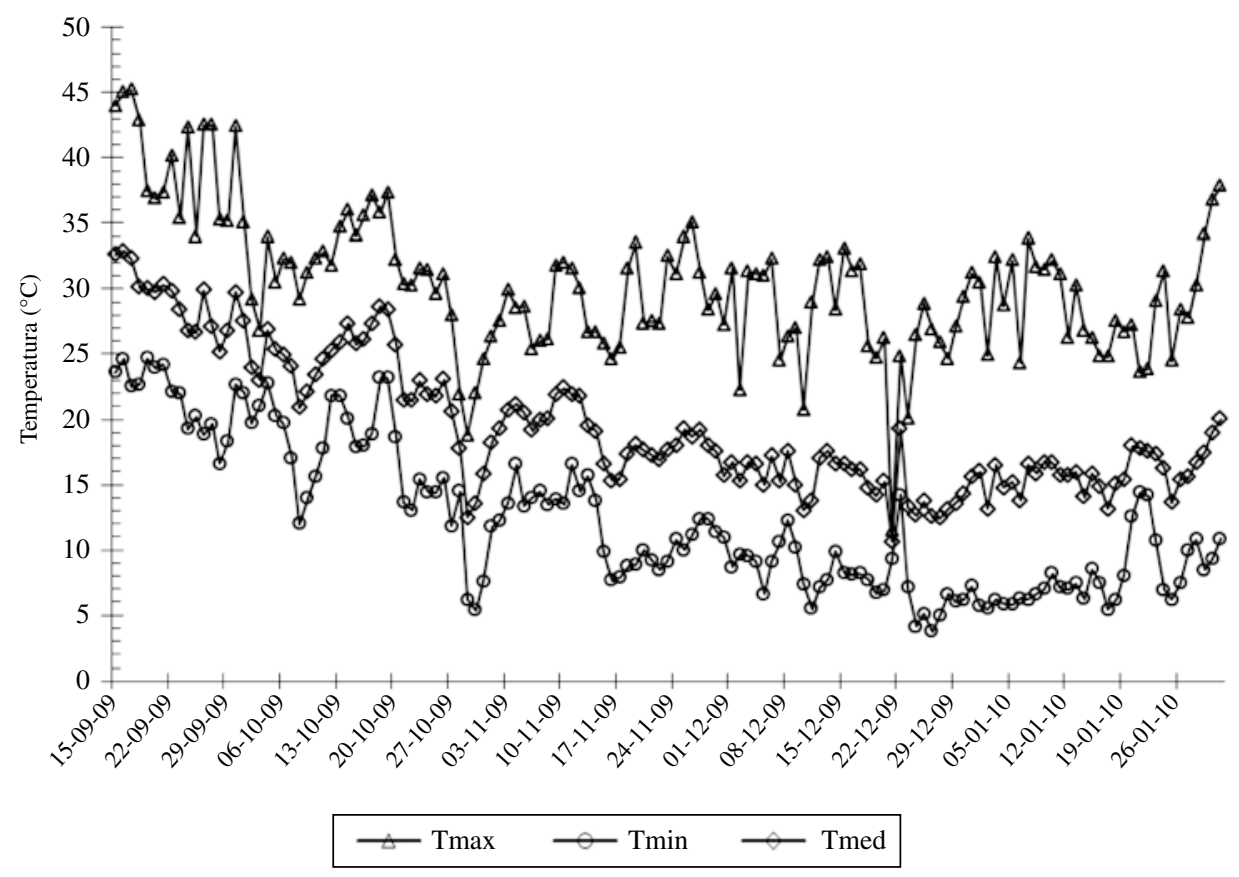

Figura 1. Comportamiento de la temperatura dentro del invernadero (Tmax: temperatura máxima, Tmin: temperatura mínima y Tmed: temperatura media). 
La longitud del fruto y firmeza de los mismos fueron similares tanto entre híbridos como entre sistemas de poda al descuelgue del cultivo, fluctuando de 22,5 a 23,5 cm y de 4,9 a 5,0 kg respectivamente, no observándose diferencias significativas entre tratamientos (Cuadros 6 y 7). Lo anterior coincide con el estudio en variedades de pepino realizado por Té (2008), quien tampoco encontró diferencias significativas para la longitud y firmeza del fruto; al igual que con Premalatha et al. (2006) quienes no encontraron diferencias para la longitud del fruto entre los sistemas de poda al descuelgue del cultivo. Asimismo, los resultados obtenidos coinciden con Wittwer y Honma (1997), al igual que Té (2008), quienes mencionan que la longitud del pepino americano fluctúa entre 20 y $25 \mathrm{~cm}$, no siendo menor de $15 \mathrm{~cm}$ (USDA, 1997).

El diámetro del fruto se vio afectado por el híbrido y por el sistema de poda al descuelgue del cultivo, observándose diferencias significativas entre tratamientos. Como se observa en el Cuadro 6, el diámetro mayor de fruto se obtuvo en el híbrido Camán con $5,1 \mathrm{~cm}$, mientras que Modán, con $4,9 \mathrm{~cm}$, presentó el menor diámetro, resultados que coinciden con el estudio realizado por Té (2008), quien encontró un diámetro promedio de $5,1 \mathrm{~cm}$ en pepino americano. En el análisis del sistema de poda al descuelgue del cultivo (Cuadro 7), Camán con descuelgue a un tallo presentó el mayor diámetro de fruto, con 5,1 cm; mientras que Esparón con descuelgue a dos tallos y Modán en ambas formas de descuelgue presentaron el menor diámetro de fruto, con promedio de $4,9 \mathrm{~cm}$. Los resultados obtenidos en Camán y Esparón coinciden con Wittwer y Honma (1997), al igual que Té (2008), quienes encontraron que el diámetro del fruto en pepino fluctuó de 5,0 a 5,7 cm, no debiendo este pasar de los 6,0 cm (USDA, 1997).

\section{Conclusiones}

En el ciclo agrícola de otoño-invierno el manejo agronómico del cultivo de pepino en invernaderos sin calefacción se puede realizar con el descuelgue del cultivo a un tallo, sin necesidad de dejar dos laterales; debido al retraso en el crecimiento de los nuevos brotes al eliminar el punto apical del tallo principal, al igual que las bajas temperaturas
Cuadro 6. Longitud $(\mathrm{cm})$, diámetro $(\mathrm{mm})$ y firmeza $(\mathrm{kg})$ del fruto en pepino (Cucumis sativus L.) bajo condiciones de invernadero.

\begin{tabular}{lccc}
\hline Tratamiento & $\begin{array}{c}\text { Longitud fruto }^{-1} \\
(\mathrm{~cm})\end{array}$ & $\begin{array}{c}\text { Diámetro fruto } \\
(\mathrm{cm})\end{array}$ & $\begin{array}{c}\text { Firmeza fruto }^{-1} \\
(\mathrm{~kg})\end{array}$ \\
\hline Camán & $22,9^{\mathrm{a}}$ & $5,1 \mathrm{a}$ & $4,6 \mathrm{a}$ \\
Esparón & $23,1^{\mathrm{a}}$ & $5,0 \mathrm{ab}$ & $4,9 \mathrm{a}$ \\
Modán & $23,5^{\mathrm{a}}$ & $4,9 \mathrm{~b}$ & $4,8 \mathrm{a}$ \\
\hline
\end{tabular}

Medias seguidas por la misma letra no son significativamente diferentes $(P=0,05)$.

Cuadro 7. Longitud $(\mathrm{cm})$, diámetro $(\mathrm{mm})$ y firmeza (kg) del fruto en pepino (Cucumis sativus $\mathrm{L}$.) bajo condiciones de invernadero.

\begin{tabular}{lccc}
\hline Tratamiento & $\begin{array}{c}\text { Longitud fruto }^{-1} \\
(\mathrm{~cm})\end{array}$ & $\begin{array}{c}\text { Diámetro fruto }^{-1} \\
(\mathrm{~cm})\end{array}$ & $\begin{array}{c}\text { Firmeza fruto }^{-1} \\
(\mathrm{~kg})\end{array}$ \\
\hline Camán $^{1}$ & $23,3^{\mathrm{a}}$ & $5,1 \mathrm{a}$ & $4,5 \mathrm{a}$ \\
Camán $^{2}$ & $22,5^{\mathrm{a}}$ & $5,0 \mathrm{ab}$ & $4,8 \mathrm{a}$ \\
Esparón $^{1}$ & $23,5^{\mathrm{a}}$ & $5,0 \mathrm{ab}$ & $4,8 \mathrm{a}$ \\
Esparón $^{2}$ & $22,8^{\mathrm{a}}$ & $4,9 \mathrm{~b}$ & $5,0 \mathrm{a}$ \\
Modán $^{1}$ & $23,5^{\mathrm{a}}$ & $4,9 \mathrm{~b}$ & $4,8 \mathrm{a}$ \\
Modán $^{2}$ & $23,5^{\mathrm{a}}$ & $4,9 \mathrm{~b}$ & $4,8 \mathrm{a}$ \\
\hline
\end{tabular}

1 representa descuelgue a un tallo y ${ }^{2}$ representa descuelgue a dos tallos.

Medias seguidas por la misma letra no son significativamente diferentes $(P=0,05)$.

retardan el crecimiento de los brotes secundarios. Además de que dicha técnica requiere de menor mano de obra.

No se observó precocidad por parte de alguno de los híbridos evaluados, al igual que por el sistema de poda al descuelgue del cultivo, iniciando la floración a los 33 días después de la siembra y la cosecha a los 69 días.

El número de frutos por planta en el híbrido Esparón, con descuelgue del cultivo a un tallo, fue mayor respecto de Camán y Modán, recomendándose su implementación por presentar también bajo porcentaje de flores masculinas y menor cuateo.

El peso del fruto, al igual que los parámetros de calidad, representados estos por la longitud, el diámetro y la firmeza del fruto, estuvieron dentro de los estándares establecidos para pepino americano, con un peso promedio de $330 \mathrm{~g}$ en la categoría Fancy, longitud de $23,2 \mathrm{~cm}$, diámetro de $5,0 \mathrm{~cm}$ y firmeza de $4,8 \mathrm{~kg}$. 


\section{Literatura Citada}

Antón, M.A

2004 Utilización del análisis del ciclo de vida en la evaluación del impacto ambiental del cultivo bajo invernadero mediterráneo. Tesis doctoral. Universidad Politécnica de Catalunya. Projectes D’Enginyeria. España.

Destenave, J.C.

2007 La producción de cultivos en invernaderos la mejor alternativa para invertir en México. El Campo Avanza. Órgano Informativo de la Secretaría de Fomento Agropecuario. 2: 9.

\section{FAOSTAT}

2010 Food and Agriculture Organization of the United Nations. Disponible en http: //faostat.fao.org/site/342/default.aspx. Consultado en Noviembre 2 de 2010.

\section{FUMIAF}

2005 Cultivo de pepino europeo en invernaderos de alta tecnología en México. Fundación Mexicana para la Investigación Agropecuaria y Forestal, A.C. Sagarpa, México. p. 37.

Garza, M.; Molina, M.

2008 Manual para la producción de tomate en invernadero en suelo en el Estado de Nuevo León. SAGARPA, México. p. 183.

Grijalva, R.L.; Robles, F.

2003 Avances en la producción de hortalizas en invernaderos. Publicación Técnica No. 7. INIFAP-CIRNO-CECAB. Caborca, Sonora. México. 14-18.

Hernández, G.

2006 Manejo del pepino en invernadero. En: Diplomado Internacional en Agricultura Protegida. Módulo 5. Cd. Obregón, Sonora, México. p. 49.

Hochmuth, R.C.

2001 Greenhouse cucumber production- Florida greenhouse vegetable production handbook. Volume 3. University of Florida. Cooperative Extension Service. Institute of Food and Agricultural Sciences. USA. p. 7.

Hochmuth, R.C.; Leon, L.L.C.; Hochmuth, G.J.

1996 Evaluation of twelve greenhouse cucumber cultivars and two training systems over two seasons in Florida. Proc. Fla. State Hort. Soc., 109: 174-177.

Kader, A.A.

1996 Maturity, ripening, and quality relationships of fruitvegetables. Acta Horiculturae, 434: 249-256.

\section{MERCANET}

2007 Situación del mercado estadounidense de pepino. Consejo Nacional de Producción. Sistema de Información e Inteligencia de Mercados. Boletín Marzo, Número 1. San José, Costa Rica. Disponible en http: //www.cnp.go.cr/ php_mysql/admin/KTML/uploads/files/boletines/Pepino marzo_2007.pdf. Consultado en Noviembre 2 de 2010.

Premalatha, M.G.S.; Wahundeniya, K.B.; Weerakkody, W.A.P.; Wicramathunga, C.K

2006 Plant training and spatial arrangement for yield improvements in greenhouse cucumber (Cucumis sativus L.) varieties. Tropical Agricultural Research, 18: 346-357.

SAS Institute Inc.

1996 The SAS System for Windows Release 6.12. Cary, N. C. USA.

SIAP

2010 Servicio de Información Agroalimentaria y Pesquera. Sagarpa, México. Disponible en http: //reportes.siap.gob. mx/Agricola_siap/ResumenProducto.do. Consultado en Noviembre 2 de 2010.

Staub, J.E.; Robbins, M.D.; Wehner, T.C.

2009 Cucumber. Cucurbit Breeding. Horticultural Science. North Carolina State University. p. 43. Disponible en http: // cuke.hort.ncsu.edu/cucurbit/wehner/articles/book15.pdf. Consultado en Noviembre 2 de 2010.

Té, E.

2008 Producción orgánica de tres variedades de pepino bajo condiciones de invernadero. Tesis de licenciatura. Universidad Autónoma de Querétaro. Facultad de Ingeniería. México.

USDA

1997 United States Standards for grades of cucumbers. United States Department of Agriculture. Agricultural Marketing Service. Fruit and Vegetable Division. Fresh Products Branch. P. 7.

Wehner, T.C.; Maynard, D.N.

2003 Cucumbers, melons, and other cucurbits. Volume 1. Encyclopedia of food and culture. New York, USA. 474-479.

Wittwer, S.H.; Honma, S.

1997 Greenhouse tomatoes, lettuce, and cucumbers. Section 3, Greenhouse cucumbers. Michigan State University, USA. Disponible en http: //www.lpl.arizona.edu/ bcohen/cucumbers/ greenhouse.html. Consultado en Noviembre 2 de 2010. 